\title{
Uso da Somatotropina Bovina Recombinante - rbST como Alternativa para a Produção de Leite de Cabra na Entressafra ${ }^{1}$
}

\author{
Paula Galvano Barbosa ${ }^{2}$, Heraldo Cesar Gonçalves ${ }^{3}$, Francisco Stefano Wechsler ${ }^{3}$, \\ Kleber Tomás de Resende ${ }^{4}$, Denise Rangel da Silva Sartori ${ }^{5}$, Laís Fernanda Callado de Pauli ${ }^{6}$, \\ Lilian Martini Pulz ${ }^{6}$, Tiago Creste Losi ${ }^{6}$
}

\begin{abstract}
RESUMO - O experimento desenvolveu-se no Setor de Caprinocultura da FMVZ - UNESP - Campus de Botucatu, com o objetivo de estudar a influência da somatotropina bovina recombinante (rbST - nome comercial: Boostin $250 \mathrm{mg}$ ) na produção de leite, nos constituintes do leite, peso e escore de condição corporal de cabras durante o ciclo lactacional. Foram usadas 21 cabras da raça Parda Alpina, com idade entre 1 1 1/2 e 4 anos, distribuídas aleatoriamente entre três tratamentos de sete animais cada: controle (T1 - sem rbST); completo, safra e entressafra (T2 - rbST após 45 dias de lactação); e apenas entressafra (T3 - rbST após 154 dias de lactação). Os animais dos três tratamentos receberam a mesma alimentação, que diferiu apenas na quantidade de concentrado, ajustada de acordo com a produção de leite dos animais. Não se observou aumento na produção de leite dos animais tratados, mas a persistência da lactação foi maior nestes animais. Não se observou diferença entre animais controles e tratados quanto aos constituintes do leite (densidade, gordura, EST, lactose e proteína). O peso e o índice de condição corporal também não apresentaram diferença significativa entre animais tratados e controle.
\end{abstract}

Palavras-chave: caprinos, curva de lactação, persistência

\section{Use of Recombinant Bovine Somatotropin - rbST as an Alternative for Lactating Goats During the Low Production Season}

\begin{abstract}
The experiment was conducted at the Goat Husbandry Sector of FMVZ - UNESP - Botucatu Campus, with the purpose of studying the influence of recombinant bovine somatotropin (rbST - commercial name: Boostin $250 \mathrm{mg}$ ) on the lactation curve, milk production, milk constituents, body weight and body score of goats during the lactation cycle. Twenty-one Alpine Brown goats between $1 \frac{1}{2}$ and 4 years old were used, randomly allotted to three treatments with seven animals each: control (T1 - no rbST), complete (T2 - rbST after the milk production peak) and low production season (T3 - rbST during the low production season). All animals received the same diet, excepting the amount of concentrate, which was adjusted according to milk yield. No increase in milk yield was observed in the treated animals, but lactation persistency was greater in these goats. The body weight and the body score also did not show significant difference between treatment and control animals.
\end{abstract}

Key Words: goats, lactation curve, persistency

\section{Introdução}

O leite de cabra apresenta particularidades que o diferencia dos demais normalmente consumidos pela população brasileira. Vale destacar a maior porcentagem de glóbulos de gordura de menor tamanho (Aguirre, 1986) e os menores teores de $\alpha$-S1-caseína (Furtado, 1983; Crosclaude et al., 1994), que lhe conferem maior digestabilidade e menor alerginicidade. Por estes motivos, o leite de cabra é considerado essencial a determinadas crianças e idosos que sofrem de intolerância ao leite de vaca.
As cabras em reprodução estacional (Mouchreck $\&$ Moulin, 1987) entram em cio quando o número de horas de luz do dia está diminuindo. Nestas condições, as cabras da região sudeste do Brasil concentram seus cios entre os meses de fevereiro e maio, ocorrendo os partos entre julho e outubro, tornando o leite um produto estacional. Dessa forma, têm-se excesso de leite entre agosto e fevereiro (safra) e falta na entressafra (março a julho). Na entressafra, os produtores conseguem melhores preços com a venda do leite, mas a produção é reduzida.

Algumas alternativas têm sido testadas para quebrar

\footnotetext{
${ }^{1}$ Parte da Dissertação de Mestrado do primeiro autor, apresentada à FMVZ, UNESP, Botucatu-SP.

2 Zootecnista, MS, FMVZ, UNESP, Botucatu.

${ }^{3}$ Professor da FMVZ, UNESP, Dep. de Prod. e Expl. Animal, Botucatu-SP. E.mail: heraldo@fca.unesp.br; wechsler@fca.unesp.br

${ }^{4}$ Professor da FCAVJ, UNESP, Dep. de Zootecnia, Jaboticabal-SP. E.mail: klebertr@fcavj.unesp.br

${ }^{5}$ Professora do IB, UNESP, Dep. de Fisiologia, Botucatu-SP. E.mail: dsartori@ibb.unesp.br

6 Discentes de Zootecnia da FMVZ, UNESP, Botucatu-SP.
} 
a estacionalidade reprodutiva das cabras. Os métodos hormonais para indução de cios férteis ainda são uma boa alternativa (Cortel et al., 1988), pois consegue-se em torno de 40 a $70 \%$ de sucesso (Cortel \& Lebouf, 1990). Esta estratégia, porém, apresenta o inconveniente de produzir em alguns animais resistência aos hormônios aplicados (Baril et al., 1996) e exigir a compra de produtos que nem sempre estão à disposição dos produtores, principalmente dos pequenos.

Outra saída normalmente empregada é a indução de cios férteis por meio da simulação de fotoperíodo, usando-se, para isso, luz artificial (Rodrigues, 1992) simultaneamente ao efeito macho. Os resultados deste método têm sido animadores, embora baseados em poucos trabalhos científicos. As dificuldades são a necessidade de energia elétrica e longo tempo para a efetivação dos procedimentos (aproximadamente 3 a 4 meses).

Uma alternativa para aumentar a produção de leite no período de entressafra seria a aplicação de hormônio de crescimento (GH), visando mudanças na curva de lactação. Estas mudanças estariam relacionadas com aumento da persistência do pico de lactação, sem alongar o período de lactação das cabras, não alterando, portanto, a época normal de parição.

Efeitos e formas de atuação da somatotropina bovina (bST)

A atuação e capacidade do hormônio de crescimento $(\mathrm{GH})$ em aumentar a produção de leite de ruminantes leiteiros estão bem estabelecidas. A maior parte da literatura que diz respeito às propriedades galactopoéticas do GH tem detalhado os efeitos em vacas, mas respostas semelhantes têm sido observadas em ovelhas e cabras. Todavia, os estudos desenvolvidos nestas duas espécies utilizam tratamentos menos prolongados (Prosser et al., 1991).

A somatotropina bovina é constituída por uma simples cadeia de polipeptídeos com 191 aminoácidos, produzida pela glândula pituitária anterior. Foi o primeiro hormônio descoberto com capacidade de aumentar o peso corporal e a agregação de proteínas na musculatura, mas em ruminantes também exerce efeito galactopoéticos.

Os mecanismos envolvidos no efeito galactopoético do GH envolvem diversos eventos que podem ser classificados em três tipos: o primeiro refere-se ao incremento na capacidade sintética da glândula mamária (Nielsen, 1988) - essa ação seria mediada por uma somatomedina mais especifica, o IGF-I, uma vez que o tecido mamário não possui receptores para a somatotropina (Prosser \& Mepham, 1989); o segundo é uma alteração, em favor da glândula mamária, na utilização de nutrientes e mobilização de tecidos não mamários, que fornecem nutrientes essenciais para a síntese do leite, principalmente tecido adiposo (McDowell et al., 1987; Soderholm et al., 1988); e o terceiro seria um aumento preferencial no fluxo sangüíneo para a glândula mamária (Mepham et al., 1984).

Mephametal.(1984), usando quatro cabras lactantes, avaliaram em 10 ocasiões da lactação o efeito do GH. Ofluxo sangüíneo mamário, nas dez ocasiões em que foi mensurado, aumentou $18,1 \%$ e nas oito ocasiões em que foi observada diferença significativa, em 19,4\%. O aumento médio na produção de leite em todas as ocasiões foi de $8,0 \%$ e nas ocasiões em que se observou diferença significativa, de $9,75 \%$. Foi observado aumento no teor de proteína (\%) em apenas uma ocasião e na produção de proteína $(\mathrm{kg})$ em cinco ocasiões.

Em outro experimento (Nielsen, 1988) com seis cabras, quatro foram submetidas ao tratamento com GH. O experimento consistiu de um período de controle $\mathrm{C} 1$ (dez dias antes do início do tratamento), tratamento T (13 dias) e controle C2 (13 dias); as outras duas cabras não receberam hormônio. Das quatro cabras que receberam somatotropina, três apresentaram aumento significativo na produção de leite de $9,6 \%$ do período $\mathrm{C} 1$ para T. Após a interrupção do tratamento, a produção de leite declinou consideravelmente nas três cabras que haviam respondido ao tratamento e em menor magnitude na cabra que não respondeu ao tratamento. Não foi observada alteração no conteúdo de gordura, proteína e lactose do leite. A maior atividade de síntese na glândula mamária ficou evidenciada pelo aumento da quantidade das enzimas acetil-CoA carboxilase e acetil-CoA sintetase em 34,6 e 26,5\%, respectivamente, em biópsia de tecido mamário (Nielsen, 1988).

Disenhaus et al. (1995) observaram que cabras tratadas com GH durante um período de quatro semanas (28 dias) apresentaram aumento médio de $28,6 \%$, em relação ao controle, na produção de leite, que chegou a $40,0 \%$ no final do período. A produção de gordura e proteína aumentou com a suplementação do $\mathrm{GH}$, em relação ao controle, em 41,6 e 23,7\%, respectivamente. A porcentagem de gordura foi maior na primeira e quarta semanas e a porcentagem de proteína foi menor na primeira semana. As cabras que receberam o bST não aumentaram a ingestão de alimentos e tão pouco foi observada diferença no

R. Bras. Zootec., v.31, n.5, p.2011-2023, 2002 
balanço energético, calculado de acordo com Sauvant \& Morand-Fehr (1991). Baldi (1999) também relata que cabras Saanen tratadas com rbST apresentaram incremento de aproximadamente $23 \%$ na produção de proteína e redução no teor de proteína de aproximadamente $1,5 \mathrm{~g} / \mathrm{kg}$ de leite. $\mathrm{O}$ autor sugere que o tratamento com rbST, em ovelhas, cabras e búfalas, aumenta a produção de leite, sem alterar suas características.

A administração de bST em cabras alpinas, em intervalos de quatro semanas, durante 12 semanas, proporcionou aumento médio na produção de leite de $300 \mathrm{~g} / \mathrm{dia}(13 \%)$. A produção de leite aumentou no dia da administração, atingiu o pico entre 3 e 5 dias e diminuiu progressivamente até a seguinte administração (Gallo et al., 1997).

Chadio et al. (2000) usaram oito cabras alpinas multíparas, após oito semanas de lactação, divididas em dois tratamentos (tratadas e não tratadas) em um delineamento em "swich-back", com três períodos de 28 dias cada. Os animais tratados receberam a cada 14 dias uma injeção subcutânea de $160 \mathrm{mg}$ de rbST. Foi observado aumento na produção de leite de 12,6\% (1506 vs. $1337 \mathrm{~mL} /$ dia), porcentagem de gordura $(3,26 \mathrm{vs} .2,90) \mathrm{e}$ porcentagem de lactose ( 3,88 vs. 3,55$)$. Não foi observada diferença no peso corporal dos animais, na contagem de células somáticas, no conteúdo de proteína (3,57 vs. 3,43) e na concentração de b-hidróxibutirato e ácidos graxos não esterificados do plasma.

Com o intuito de apresentar nova alternativa para o problema da estacionalidade produtiva dos caprinos, este trabalho foi conduzido com o objetivo de estudar a influência da somatotropina bovina recombinante (rbST - nome comercial: Boostin $250 \mathrm{mg}$ ) na curva de lactação, produção de leite, nos constituintes do leite, no peso corporal e no escore de condição corporal de cabras leiteiras durante o ciclo lactacional. Também foi realizada uma avaliação econômica simplificada dos tratamentos, levando em consideração o consumo de ração, o custo do bST e o preço do leite na safra e entressafra. Além disso, foi possível avaliar as características reprodutivas das cabras após o término do experimento.

\section{Material e Métodos}

O experimento foi realizado no Setor de Caprinocultura da Faculdade de Medicina Veterinária e Zootecnia, UNESP, Câmpus de Botucatu, localizado na Fazenda Experimental Lageado, no período de maio de 1999 a agosto de 2000.
Foram utilizadas 21 cabras da raça Parda Alpina, com idade entre 1 1/2 e 4 anos. Dessa forma, havia animais de primeira, segunda, terceira e quarta lactações. As cabras foram acasaladas a partir do mês de maio de 1999 e os partos ocorreram a partir do mês de outubro do mesmo ano.

Os animais foram distribuídos, aleatoriamente após o parto, em três grupos de sete animais. $\mathrm{Na}$ Tabela 1, observa-se a distribuição dos animais dentro dos tratamentos nas diferentes ordens de lactação. Cada grupo ficou em uma baia coberta, com piso ripado suspenso, com livre acesso ao solário.

Durante todo o período experimental, forneceu-se volumoso duas vezes ao dia, à base de capim-elefante (Pennisetum purpurem Schum) picado ad libitum, e feno de coastcross (Cynodon Dactilon) controlado, suficiente para atender as necessidades de mantença. O capim-elefante utilizado no experimento foi retirado de uma capineira pertencente à Fazenda Lageado, entretanto, como também era de uso de outros setores experimentais, não foi suficiente, precisando no final do experimento utilizar capim da beira do riacho que corta a fazenda. Nesse ano, houve longo um período de seca (o capim estava de má qualidade) e nos meses de janeiro e fevereiro de 2000, um longo período de chuva, o que melhorou a qualidade do capim.

O concentrado foi fornecido três vezes ao dia de acordo com a produção de leite (NRC, 1981), na proporção de $1 \mathrm{~kg}$ de concentrado para cada 2,5 litros de leite produzido, com um adicional de $20 \%$ para incrementar a produção. O ajuste da

Tabela 1 - Número de animais por tratamentos experimentais nas diferentes ordens de lactação

Table 1 - Number of animals per treatment, according to lactation order

\begin{tabular}{lccc}
\hline $\begin{array}{l}\text { Ordem de lactação } \\
\text { Lactation } \text { order }\end{array}$ & \multicolumn{3}{c}{$\begin{array}{c}\text { Tratamentos experimentais } \\
\text { Experimental treatments }\end{array}$} \\
\cline { 2 - 4 } & $\mathrm{T} 1$ & $\mathrm{~T} 2$ & $\mathrm{~T} 3$ \\
\hline $\begin{array}{l}\text { Primeira } \\
\text { First }\end{array}$ & 1 & 4 & 4 \\
$\begin{array}{l}\text { Segunda } \\
\text { Second } \\
\begin{array}{l}\text { Terceira } \\
\text { Third }\end{array}\end{array}$ & 3 & 1 & 1 \\
$\begin{array}{l}\text { Quarta } \\
\text { Fourth }\end{array}$ & 2 & 0 & 0 \\
\hline
\end{tabular}

1 Tratamentos experimentais: $\mathrm{T} 1$ = tratamento controle; $\mathrm{T} 2$ = tratamento completo; $\mathrm{T} 3=$ tratamento de entressafra.

${ }^{1}$ Experimental treatments: $T 1=$ control; $T 2=$ full treatment; $T 3=10 \mathrm{~W}$ production season.

R. Bras. Zootec., v.31, n.5, p.2011-2023, 2002 
quantidade de concentrado foi feito semanalmente. Água e mistura mineral foram consumidos à vontade.

A composição bromatológica dos alimentos está apresentada na Tabela 2; a composição do volumoso (capim-elefante), na Tabela 3; e a composição percentual dos ingredientes do concentrado, na Tabela 4 . O teor de matéria seca foi determinado após secagem do material em estufa a $105^{\circ} \mathrm{C}$; a proteína bruta $(\mathrm{PB})$, pelo método micro Kjeldahl; e os teores de fibra bruta (FB), fibra detergente neutro (FDN), fibra detergente ácido (FDA) e extrato etéreo (EE), segundo metodologia descrita por Silva (1990). Os nutrientes digestíveis totais (NDT) foram estimados de acordo com Weiss et al. (1992).

Foram utilizadas 21 cabras distribuídas em três tratamentos experimentais:

$\mathrm{T} 1 \Rightarrow$ tratamento controle: sete animais que não receberam tratamento hormonal.

$\mathrm{T} 2 \Rightarrow$ tratamento hormonal completo: sete animais receberam tratamento hormonal, supostamente após o pico de lactação (45 dias após o parto), até o final do período experimental.

Tabela 2 - Composição bromatológica dos alimentos em percentual da matéria seca Table 2 - Chemical composition of feedstuffs (as dry matter basis)

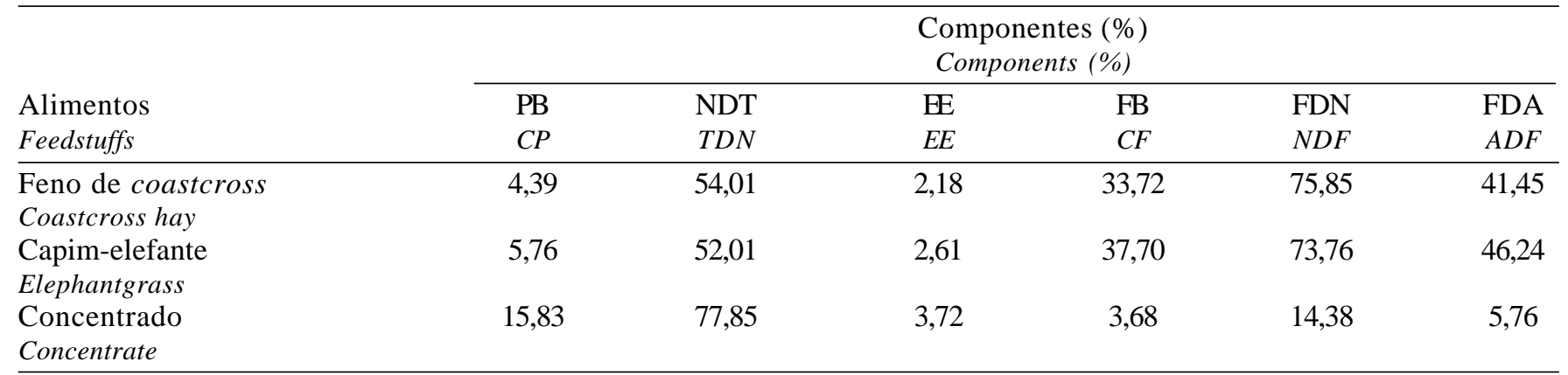

$\mathrm{PB}=$ proteína bruta; NDT = nutrientes digestíveis totais; $\mathrm{EE}$ = extrato etéreo; FB = fibra bruta, FDN = fibra em detergente neutro; FDA = fibra em detergente ácido.

$C P=$ crude protein; $T D N=$ total digestible nutrients; $E E=$ ether extract; $C F=$ crude fiber, $N D F=$ neutral detergent fiber; $A D F=$ acid detergent fiber.

Tabela 3 - Composição bromatológica (\%) do capim-elefante, em função das colheitas Table 3 - Chemical composition (\%) of elephantgrass, according to the harvest time

\begin{tabular}{|c|c|c|c|c|c|}
\hline $\begin{array}{l}\text { Colheita } \\
\text { Sampling }\end{array}$ & $\begin{array}{l}\mathrm{PB} \\
C P\end{array}$ & $\begin{array}{l}\text { NDT } \\
T D N\end{array}$ & $\begin{array}{l}\text { FB } \\
C F\end{array}$ & $\begin{array}{l}\text { FDN } \\
N D F\end{array}$ & $\begin{array}{l}\text { FDA } \\
A D F\end{array}$ \\
\hline $\begin{array}{l}\text { Dezembro/99 } \\
\text { December/00 }\end{array}$ & 2,56 & 51,43 & 39,01 & 76,50 & 47,40 \\
\hline $\begin{array}{l}\text { Janeiro/00 } \\
\text { January/00 }\end{array}$ & 7,28 & 51,39 & 34,46 & 68,82 & 44,19 \\
\hline $\begin{array}{l}\text { Fevereiro/00 } \\
\text { February/00 }\end{array}$ & 10,90 & 55,31 & 35,03 & 70,61 & 42,26 \\
\hline $\begin{array}{l}\text { Março/00 } \\
\text { March/00 }\end{array}$ & 3,45 & 49,89 & 42,74 & 80,55 & 52,40 \\
\hline $\begin{array}{l}\text { Abril/00 } \\
\text { April/00 }\end{array}$ & 4,60 & 52,93 & 37,24 & 73,89 & 44,34 \\
\hline $\begin{array}{l}\text { Maio/00 } \\
\text { May/00 }\end{array}$ & 4,03 & 51,50 & 39,75 & 73,39 & 47,29 \\
\hline $\begin{array}{l}\text { Junho/00 } \\
\text { June/00 }\end{array}$ & 2,85 & 51,17 & 38,91 & 73,69 & 47,62 \\
\hline $\begin{array}{l}\text { Julho/00 } \\
\text { June/00 }\end{array}$ & 4,44 & 53,83 & 34,18 & 70,77 & 39,98 \\
\hline $\begin{array}{l}\text { Agosto/00 } \\
\text { August/00 }\end{array}$ & 6,24 & 50,61 & 36,15 & 75,70 & 50,71 \\
\hline
\end{tabular}

R. Bras. Zootec., v.31, n.5, p.2011-2023, 2002 
Tabela 4 - Composição do concentrado em percentual da matéria natural

Table 4 - Concentrate composition (as fed)

\begin{tabular}{lc}
\hline $\begin{array}{l}\text { Ingredientes } \\
\text { Ingredients }\end{array}$ & $\begin{array}{c}\text { Porcentagem } \\
\text { Percentage }\end{array}$ \\
\hline $\begin{array}{l}\text { Milho } \\
\text { Corn }\end{array}$ & 74,00 \\
$\begin{array}{l}\text { Farelo de trigo } \\
\text { Wheat bran }\end{array}$ & 6,00 \\
$\begin{array}{l}\text { Farelo de soja } \\
\text { Soybean meal }\end{array}$ & 17,00 \\
Calcário \\
$\begin{array}{l}\text { Limestone } \\
\text { Mistura mineral (Fosbovi 20) } \\
\text { Mineral salt }\end{array}$ \\
\hline
\end{tabular}

T3 $\Rightarrow$ tratamento de entressafra; sete animais receberam tratamento hormonal no início do período de entressafra (154 dias após o parto, mês de março) até o final do período experimental.

O tratamento hormonal consistiu em injeções de $70 \mathrm{mg}$ de Somatotropina Bovina Recombinante (rbST) de liberação lenta $(0,56 \mathrm{~mL}$ de BOOSTIN $250 \mathrm{mg}$ - COOPERS) a cada 14 dias, por via subcutânea, na fossa ísqueo-retal previamente desinfetada com álcool iodado, alternando-se os lados esquerdo e direito, a cada aplicação. Após a aplicação, o local era suavemente massageado. Essa dosagem baseou-se na utilizada por Disenhaus et al. (1995) de $5 \mathrm{mg} / \mathrm{dia}$ (o que corresponde a $1 / 4$ da dose recomendada para bovinos).

Consta na Figura 1 o esquema das atividades realizadas durante o período experimental.

\section{Peso e índice de condição corporal}

Os animais foram pesados mensalmente, pela manhã, em balança com capacidade de $300 \mathrm{~kg}$ e divisões de 100 g. Foram feitas 11 observações (Figura 1).

Os animais, após serem pesados, foram avaliados quanto ao índice de condição corporal, utilizando-se o procedimento do Institut Nacional de la Recherche Agronomique - INRA (1990), que é o mesmo utilizado por Morand-Fehr \& Herviev (1989). Dessa forma, também foram feitas 11 observações para índice de condição corporal (Figura 1).

\section{Produção de leite}

Foram realizadas duas ordenhas diárias, às 7h30 e às $15 \mathrm{~h}$. O controle leiteiro foi realizado a cada 14 dias, usando-se um dinamômetro com precisão de $100 \mathrm{~g}$. Foram feitas 19 mensurações no total (Figura 1).

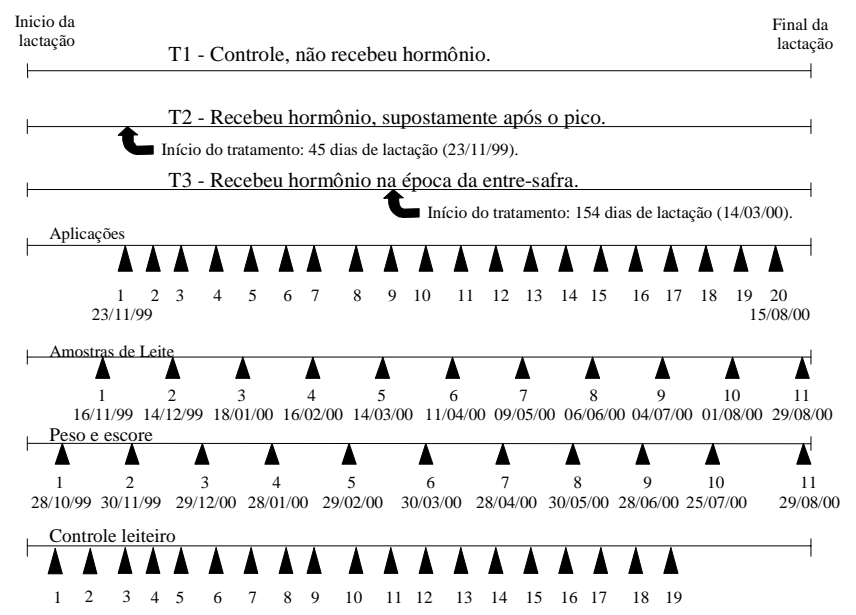

Figura 1 - Esquema dos tratamentos experimentais e cronograma das atividades.

Figure 1 - Outline of the experimental treatments and chronogram of activities.

O controle leiteiro foi feito até o dia 29/08/2000, mas alguns animais já haviam secado espontaneamente. Por este motivo, optou-se por utilizar o último controle leiteiro que estivesse completo (com todos os animais - 04/07/2000).

\section{Constituintes do leite}

Foram coletadas amostras mensais de leite de apenas cinco animais por tratamento (Figura 1); estes animais eram sempre os mesmos. Essas amostras foram compostas usando-se uma amostra da ordenha da manhã e uma amostra da ordenha da tarde. As seguintes características foram avaliadas: densidade, extrato seco total (EST), proteína bruta e lactose, segundo a AOAC (1975), e matéria graxa (gordura), segundo o Instituto Adolfo Lutz (1985). Estas análises foram feitas no Laboratório do Departamento de Tecnologia de Alimentos da Faculdade de Medicina Veterinária e Zootecnia - UNESP - Campus de Botucatu (Lageado).

Não foi feito controle do consumo alimentar dos animais do experimento, em função de os animais do mesmo tratamento estarem em uma mesma baia e do tipo de alimentação oferecido.

\section{Análises e procedimentos estatísticos}

O delineamento utilizado foi o de medidas repetidas, de acordo com o modelo matemático: $\mathrm{Y}_{\mathrm{ijk}}=\mathrm{u}+\mathrm{T}_{\mathrm{i}}+(\mathrm{C} / \mathrm{T})_{\mathrm{ij}}+\mathrm{CO}_{\mathrm{k}}+(\mathrm{T} * \mathrm{CO})_{\mathrm{ik}}+[(\mathrm{C} / \mathrm{T}) * \mathrm{CO}]_{\mathrm{ijk}}$ em que $Y_{i j k}=$ variáveis dependentes: produção de 
leite, peso, índice de condição corporal e constituintes do leite (densidade, extrato seco total, porcentagem de gordura, lactose e proteína do leite); $\mathrm{u}=$ constante; $\mathrm{T}_{\mathrm{i}}=$ efeito do tratamento $\mathrm{i}, \operatorname{com} \mathrm{i}=1,2$ e $3 ;(\mathrm{C} / \mathrm{T})_{\mathrm{ij}}=$ efeito da cabra $\mathrm{j}, \mathrm{com} \mathrm{j}=1,2, \ldots, 7$ dentro de tratamento $\mathrm{i}$ e $\mathrm{j}=1,2, \ldots 5$ para constituintes do leite, ou erro A; $\mathrm{CO}_{\mathrm{K}}=$ efeito da colheita $\mathrm{k}$, com $\mathrm{k}=1,2, \ldots, 19$ para produção de leite e $\mathrm{k}=1,2, \ldots, 11$ para peso, índice de condição corporal e constituintes do leite; $\left(\mathrm{T}^{*} \mathrm{CO}\right)_{\mathrm{ik}}=$ efeito da interação tratamento i e colheita $\mathrm{k} ;(\mathrm{C} / \mathrm{T}) * \mathrm{CO}_{\mathrm{ijk}}=$ efeito da interação com colheita $\mathrm{k}$, ou erro B.

Utilizou-se o procedimento parcelas subdivididas do programa SAEG - Sistema para Análise Estatística e Genética (UFV, 1997) para a análise.

De posse das médias de mínimos quadrados da produção de leite da interação tratamento x colheita, estas foram ajustadas pela função gama (Wood, 1967), expressa como:

$$
\mathrm{Y}_{\mathrm{t}}=\mathrm{at}{ }^{\mathrm{b}} \exp (-\mathrm{ct})
$$

em que: $Y_{t}$ é a média diária no tempo t e a, b, c, as constantes.

Utilizou-se o procedimento REGREGN do programa SAEG - Sistema para Análise Estatística e Genética (UFV, 1997), que usa o método de GaussNewton, para o ajustamento das curvas de produção de leite.

\section{Resultados e Discussão}

\section{Peso e índice de condição corporal}

O resumo da análise de variância de peso e índice de condição corporal é apresentado na Tabela 5. Não foi possível detectar efeito de tratamento $(\mathrm{P}>0,05)$ para peso e índice de condição corporal. Para o peso corporal observou-se efeito $(\mathrm{P}<0,05)$ de coleta e interação entre tratamento $\mathrm{x}$ coleta.

As médias da interação tratamento $\mathrm{x}$ coleta são apresentadas na Tabela 6. Pelo teste Tukey, corrigido para número de graus de liberdade e variância, de acordo com Satterthwaite (Gomes, 1982), não foi observada diferença significativa entre as médias de tratamento dentro de coleta.

O peso médio dos animais apresentou variação semelhante entre os tratamentos ao longo das coletas (Tabela 6; Figura 2). Possivelmente, o efeito significativo da interação deva-se ao efeito das coletas dentro de um tratamento específico.

Pode-se verificar na Figura 2 que os três tratamentos obtiveram semelhante comportamento quanto ao peso corporal durante o período experimental. Isso demonstra que a qualidade da alimentação destes animais influenciou os resultados deste experimento.

A coleta 5 para peso coincide com a análise do capim-elefante de fevereiro, em que houve maior teor de proteína e menor de fibra, ou seja, melhor qualidade.

Não foi possível detectar diferenças entre os tratamentos experimentais quanto ao peso corporal dos animais.

Segundo Disenhaus et al. (1995), as oscilações que ocorrem nos pesos após as aplicações hormonais indicam mobilização de reservas corporais de lipídeos, pois obtiveram aumento na concentração de ácidos graxos não esterificados (NEFA, ou ácidos graxos livres - AGL) em cabras tratadas com bST, comparadas às do controle. Esses autores sugerem que estas variações refletem a adaptação dos ruminantes em lactação ao aumento da demanda de nutrientes para cobrir o aumento na produção de leite, sem, no

Tabela 5 - Resumo da análise de variância de peso e índice de condição corporal Table 5 - Summary of the analysis of variance for body weight and body condition score

\begin{tabular}{lccc}
\hline $\begin{array}{l}\text { Fonte de variação } \\
\text { Source of variation }\end{array}$ & $\begin{array}{c}\text { Graus de liberdade } \\
\text { Degrees of freedom }\end{array}$ & \multicolumn{2}{c}{ Quadrados médios squares } \\
\cline { 3 - 4 } & & $\begin{array}{c}\text { Peso } \\
\text { Weight }\end{array}$ & $\begin{array}{c}\text { Índice } \\
\text { Score }\end{array}$ \\
\hline $\begin{array}{l}\text { Tratamento } \\
\text { Treatment }\end{array}$ & 2 & 8,021602 & 0,168831 \\
$\begin{array}{l}\text { Erro(A) } \\
\text { Error (A) }\end{array}$ & 18 & 259,3293 & 0,265512 \\
$\begin{array}{l}\text { Coleta } \\
\text { Measurement }\end{array}$ & 10 & $122,6239^{* *}$ & $2,664935^{* *}$ \\
$\begin{array}{l}\text { Tratamento x Coleta } \\
\text { Treatment } x \text { Measurement }\end{array}$ & 20 & $10,79565^{* *}$ & 0,097403 \\
$\begin{array}{l}\text { Erro(B) } \\
\text { Error (B) }\end{array}$ & 180 & 4,501365 & 0,095671 \\
\end{tabular}

R. Bras. Zootec., v.31, n.5, p.2011-2023, 2002 
Tabela 6 - Médias de mínimos quadrados do peso corporal em função da interação tratamento e colheita

Table 6 - Least squares means of body weight, according to the treatment $x$ measurement combination

\begin{tabular}{lccc}
\hline $\begin{array}{l}\text { Coleta } \\
\text { Measurement }\end{array}$ & \multicolumn{3}{c}{$\begin{array}{c}\text { Tratamentos } \\
\text { Treatments }\end{array}$} \\
\cline { 2 - 4 } & $\mathrm{T} 1$ & $\mathrm{~T} 2$ & $\mathrm{~T}$ \\
\hline 1 & $51,34^{\mathrm{A}}$ & $53,04^{\mathrm{A}}$ & $53,90^{\mathrm{A}}$ \\
2 & $52,61^{\mathrm{A}}$ & $53,77^{\mathrm{A}}$ & $53,70^{\mathrm{A}}$ \\
3 & $50,49^{\mathrm{A}}$ & $49,43^{\mathrm{A}}$ & $51,69^{\mathrm{A}}$ \\
4 & $53,40^{\mathrm{A}}$ & $52,70^{\mathrm{A}}$ & $51,56^{\mathrm{A}}$ \\
5 & $55,79^{\mathrm{A}}$ & $55,53^{\mathrm{A}}$ & $54,49^{\mathrm{A}}$ \\
6 & $50,99^{\mathrm{A}}$ & $54,21^{\mathrm{A}}$ & $53,07^{\mathrm{A}}$ \\
7 & $51,71^{\mathrm{A}}$ & $52,83^{\mathrm{A}}$ & $50,14^{\mathrm{A}}$ \\
8 & $51,70^{\mathrm{A}}$ & $52,61^{\mathrm{A}}$ & $50,01^{\mathrm{A}}$ \\
9 & $51,21^{\mathrm{A}}$ & $52,27^{\mathrm{A}}$ & $51,84^{\mathrm{A}}$ \\
10 & $57,44^{\mathrm{A}}$ & $56,20^{\mathrm{A}}$ & $56,71^{\mathrm{A}}$ \\
11 & $60,57^{\mathrm{A}}$ & $58,11^{\mathrm{A}}$ & $56,20^{\mathrm{A}}$ \\
\hline
\end{tabular}

${ }^{1}$ Média na linha seguida de letra distinta difere $(P<0,05)$ das demais pelo teste Tukey.

${ }^{1}$ Means, within a row, followed bydifferent letters differ $(P<.05)$, according to Tukey test.

entanto, trazer problemas à saúde dos animais, pelo menos em um curto período de aplicação.

O aumento de peso observado a partir da coleta 9 deve-se ao fato de as cabras estarem em gestação, pois foram cobertas a partir do mês de maio de 2000.

Para o índice de condição corporal (ICC) foi observado efeito significativo de coleta (Tabela 5). As médias de ICC para as diferentes coletas estão apresentadas na Figura 3 e Tabela 7.

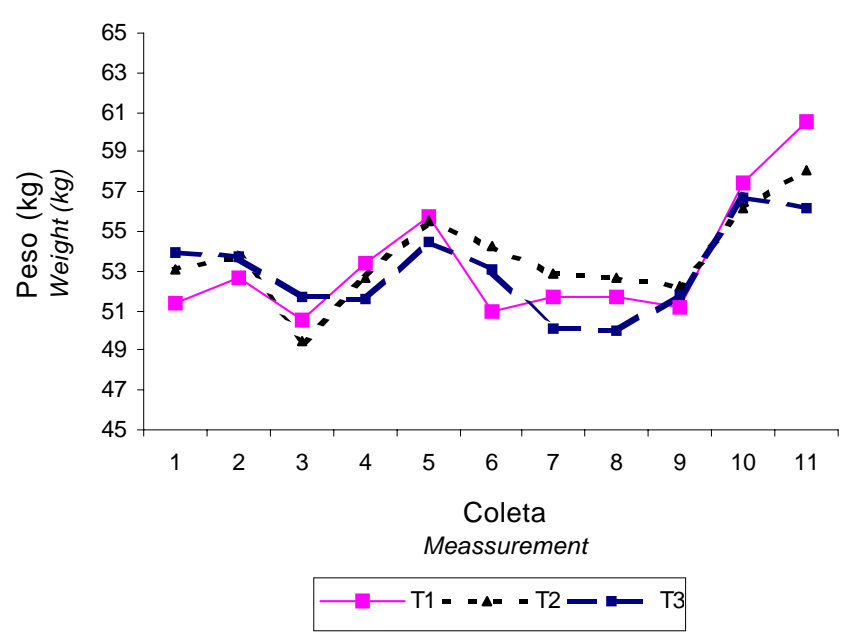

Figura 2 - Médias observadas de peso corporal por tratamento, em função de colheita.

Figure 2 - Body weight means, according to the treatment and measurement.

R. Bras. Zootec., v.31, n.5, p.2011-2023, 2002
Na coleta 5 (feita no mês de fevereiro), houve um aumento no ICC, possivelmente em virtude da melhoria do volumoso (capim-elefante), que apresentou maiores teores de proteína total e NDT (Tabela 3) nessa época.

De maneira geral, o resultado deste trabalho concorda com os obtidos por Chadio et al. (2000), que não obtiveram diferença significativa no peso corporal entre o grupo controle e o tratado.

Produção de leite

O resumo da análise de variância da produção de leite é apresentado na Tabela 8.

A coleta influenciou a produção de leite $(\mathrm{P}<0,01)$. De maneira geral, a produção de leite aumentou até a coleta 4 e, a partir daí, sofreu pequenas oscilações, tendendo a diminuir nas colheitas sucessivas.

A interação tratamento $x$ coleta apresentou resposta significativa $(\mathrm{P}<0,10)$ (Tabela 8$)$.

As médias da interação tratamento $\mathrm{x}$ coleta estão apresentadas na Tabela 9 e Figura 4.

$\mathrm{O}$ tratamento $\mathrm{T} 2$ apresentou, de maneira geral, média mais baixa, indicando que os animais deste tratamento possivelmente apresentavam um potencial genético para produção de leite menor que o dos outros dois tratamentos. Mesmo assim, responderam ao tratamento com rbST, pois conseguiram aproximar suas produções às do grupo controle (T1) e do tratamento $\mathrm{T} 3$, ao longo das coletas (Figura 4). Contudo, não houve diferença significativa entre os tratamentos para a produção de leite, com exceção das coletas 3 e 7 (Tabela 9).

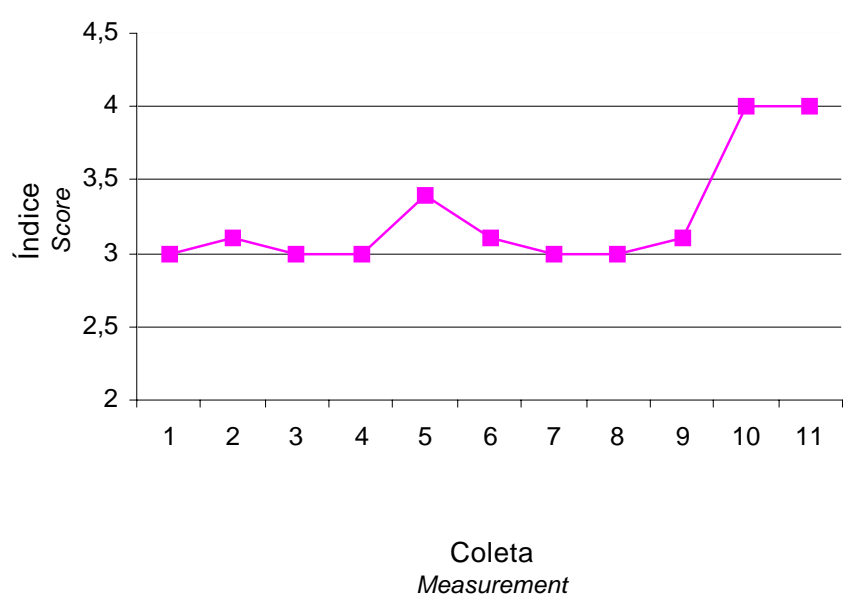

Figura 3 - Índice de condição corporal, em função da coleta.

Figure 3 - Body condition score, according to the measurement. 
Tabela 7 - Médias de mínimos quadrados do índice de condição corporal, em função da coleta

Table 7 - Least squares means of body condition score, according to the measurement

\begin{tabular}{cc}
\hline $\begin{array}{c}\text { Coleta } \\
\text { Measurement }\end{array}$ & $\begin{array}{c}\text { Índice } \\
\text { Score }\end{array}$ \\
\hline 1 & $3,0^{\mathrm{c} 1}$ \\
2 & $3,1^{\mathrm{bc}}$ \\
3 & $3,0^{\mathrm{c}}$ \\
4 & $3,0^{\mathrm{c}}$ \\
5 & $3,4^{\mathrm{b}}$ \\
6 & $3,1^{\mathrm{bc}}$ \\
7 & $3,0^{\mathrm{c}}$ \\
8 & $3,0^{\mathrm{c}}$ \\
9 & $3,1^{\mathrm{bc}}$ \\
10 & $4,0^{\mathrm{a}}$ \\
11 & $4,0^{\mathrm{a}}$ \\
\hline${ }^{1}$ Média seguida de letra distinta difere das demais $(\mathrm{P}<0,05)$ pelo \\
teste Tukey. \\
${ }^{1}$ Means within a row followed bydifferent letters differ $(P<.05)$ according \\
to Tukey test. \\
Produção de leite (Milk production).
\end{tabular}

Na coleta 3, o T3 apresentou média de produção de leite maior que o T2, e este não diferiu do T1 (Tabela 9).

Na coleta 7, o T1 apresentou média de produção de leite maior que o T2, e este não diferiu do T3 (Tabela 9).

O tratamento $\mathrm{T} 3$ passou a receber injeções de rbST a partir da coleta 11, quando sua produção voltou a aumentar, tendendo a ser maior que os outros dois tratamentos, mas esta diferença não foi significativa (Tabela 9).

Na Figura 4, pode-se observar aumento na produção de leite do T3 a partir da coleta 11, quando se iniciou o tratamento com rbST, e esse aumento persistiu até o final do experimento, embora não tenha sido constatada diferença significativa. Esse aumento pode ser devido, em parte, à melhoria da qualidade do volumoso (capim-elefante) a partir desse ponto, conforme observado na Tabela 3, e à ação do rbST. Isso vem mostrar que o rbST teve melhor eficiência quando aplicado após o meio da lactação, que coincide com o período de entressafra (Tabela 9), corroborando o observado por Gallo et al. (1997), que constataram maior envolvimento do hormônio de crescimento envolvido na proliferação de células da glândula mamária a partir do meio da lactação.

O aumento na produção de leite de cabras tratadas com rbST tem se mostrado consistente na literatura. Esse aumento é da ordem de 8 a 28,6\% (Mepham et al., 1984; Nielsen, 1988; Disenhaus et al., 1995; Gallo
Tabela 8 - Resumo da análise de variância da produção de leite

Table 8 - Summary of the analysis of variance for milk production

\begin{tabular}{lcc}
\hline $\begin{array}{l}\text { Fontes de } \\
\text { variação } \\
\begin{array}{l}\text { Source of } \\
\text { variation }\end{array}\end{array}$ & $\begin{array}{c}\text { Graus de } \\
\text { liberdade } \\
\text { Degrees } \\
\text { offreedom }\end{array}$ & $\begin{array}{c}\text { Quadrado } \\
\text { médio } \\
\text { Mean } \\
\text { square }\end{array}$ \\
\hline $\begin{array}{l}\text { Tratamento } \\
\text { Treatment } \\
\text { Erro }(\mathrm{A})\end{array}$ & 2 & 2,940108 \\
$\begin{array}{l}\text { Error }(\mathrm{A}) \\
\text { Coleta }\end{array}$ & 18 & 2,890728 \\
$\begin{array}{l}\text { Measurement } \\
\text { Tratamento x Coleta }\end{array}$ & 18 & $1,437194^{* *}$ \\
$\begin{array}{l}\text { Treatment } x \text { Measurement } \\
\text { Erro }(\mathrm{B})\end{array}$ & 36 & $0,082366^{*}$ \\
Error $(B)$ & 324 & 0,056589 \\
** $(\mathrm{P}<0,01)$. & & \\
$* *(\mathrm{P}<0,10)$. & & \\
\end{tabular}

et al., 1997; Chadio et al., 2000). No presente estudo, o aumento na produção de leite pode não ter sido estatisticamente constatado, em decorrência do menor potencial de produção das cabras do T2 e da menor quantidade de rbST administrada em comparação ao trabalho de Chadio et al. (2000), que administraram mais que o dobro da dose usada neste trabalho.

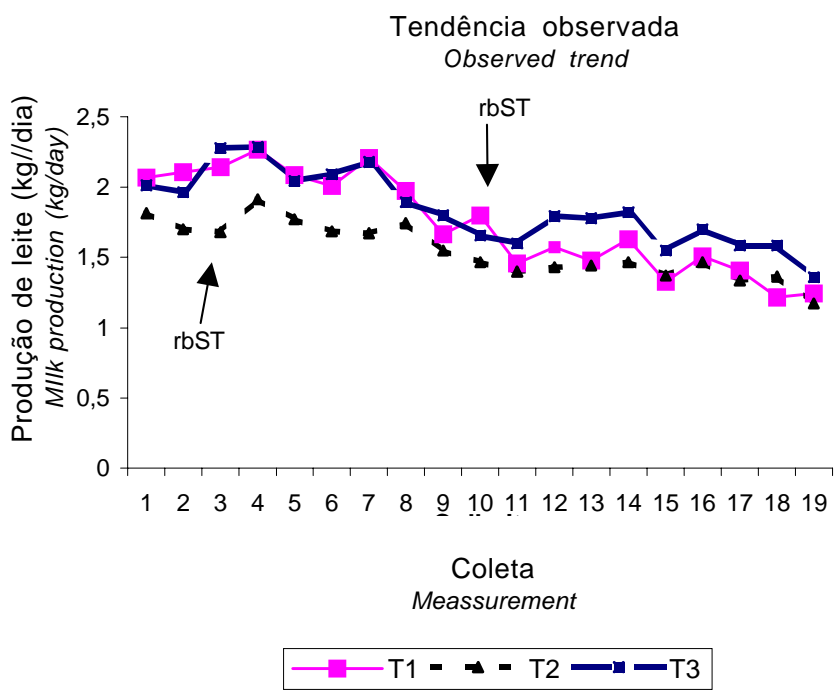

Figura 4 - Tendência observada (média de mínimos quadrados) da produção de leite por tratamento, em função da coleta.

Figure 4 - Observed trend (least squares means) of the milk production, according to the measurement. 
Tabela 9 - Média de mínimos quadrados da produção diária de leite em função da interação entre tratamento e colheita

Table 9 - Least squares means of daily milk production according to the treatment by measurement combination

\begin{tabular}{|c|c|c|c|}
\hline \multirow[t]{2}{*}{$\begin{array}{l}\text { Colheita } \\
\text { Measurement }\end{array}$} & \multicolumn{3}{|c|}{$\begin{array}{c}\text { Tratamentos } \\
\text { Treatments }\end{array}$} \\
\hline & $\mathrm{T} 1$ & $\mathrm{~T} 2$ & T3 \\
\hline 1 & $2,0678^{\mathrm{A} 1}$ & $1,8143^{\mathrm{A}}$ & $2,0143^{\mathrm{A}}$ \\
\hline 2 & $2,1071^{\mathrm{A}}$ & $1,7000^{\mathrm{A}}$ & $1,9643^{\mathrm{A}}$ \\
\hline 3 & $2,1429^{\mathrm{AB}}$ & $1,6786^{\mathrm{B} 2}$ & $2,2786^{\mathrm{A}}$ \\
\hline 4 & $2,2643^{\mathrm{A}}$ & $1,9143^{\mathrm{A}}$ & $2,2857^{\mathrm{A}}$ \\
\hline 5 & $2,0857^{\mathrm{A}}$ & $1,7714^{\mathrm{A}}$ & $2,0429^{A}$ \\
\hline 6 & $2,0071^{\mathrm{A}}$ & $1,6857^{\mathrm{A}}$ & $2,0929^{\mathrm{A}}$ \\
\hline 7 & $2,2071^{\mathrm{A}}$ & $1,6714^{\mathrm{B}}$ & $2,1786^{\mathrm{AB}}$ \\
\hline 8 & $1,9714^{\mathrm{A}}$ & $1,7429^{\mathrm{A}}$ & $1,8929^{\mathrm{A}}$ \\
\hline 9 & $1,6643^{\mathrm{A}}$ & $1,5500^{\mathrm{A}}$ & $1,8000^{\mathrm{A}}$ \\
\hline 10 & $1,8000^{\mathrm{A}}$ & $1,4643^{\mathrm{A}}$ & $1,6571^{\mathrm{A}}$ \\
\hline 11 & $1,4571^{\mathrm{A}}$ & $1,4000^{\mathrm{A}}$ & $1,6000^{\mathrm{A}}$ \\
\hline 12 & $1,5714^{\mathrm{A}}$ & $1,4286^{\mathrm{A}}$ & $1,7929^{\mathrm{A}}$ \\
\hline 13 & $1,4786^{\mathrm{A}}$ & $1,4429^{\mathrm{A}}$ & $1,7786^{\mathrm{A}}$ \\
\hline 14 & $1,6286 \mathrm{~A}$ & $1,4643^{\mathrm{A}}$ & $1,8214^{\mathrm{A}}$ \\
\hline 15 & $1,3286 \mathrm{~A}$ & $1,3714^{\mathrm{A}}$ & $1,5500^{\mathrm{A}}$ \\
\hline 16 & $1,5071^{\mathrm{A}}$ & $1,4643^{\mathrm{A}}$ & $1,7000^{\mathrm{A}}$ \\
\hline 17 & $1,4071^{\mathrm{A}}$ & $1,3357^{\mathrm{A}}$ & $1,5857^{\mathrm{A}}$ \\
\hline 18 & $1,2157^{\mathrm{A}}$ & $1,3643^{\mathrm{A}}$ & $1,5857^{\mathrm{A}}$ \\
\hline 19 & $1,2429^{\mathrm{A}}$ & $1,1714^{\mathrm{A}}$ & $1,3571^{\mathrm{A}}$ \\
\hline
\end{tabular}

${ }^{1}$ Média na mesma linha seguida de letra distinta difere das demais $(P<0,10)$ pelo teste Tukey.

${ }^{2}$ Médias em negrito marcam o início das aplicações hormonais de T2 e T3.

${ }^{1}$ Means within a row followed bydifferent letters differ $(P<.10)$, according to Tukey test.

${ }^{2}$ Bold face means mark the beginning of hormone applications in T2 and $T 3$.

As curvas de produção de leite por tratamento, ajustadas pela função gama, são apresentadas na Figura 5. As constantes para cada curva são apresentadas na Tabela 10 e as médias ajustadas por coleta, na Tabela 11.

Observando-se as curvas apresentadas na Figura 5, nota-se que, na curva do T1, que não recebeu tratamento hormonal, a produção declinou acentuadamente em relação aos tratamentos $\mathrm{T} 2 \mathrm{e}$ T3. Na Tabela 11 são apresentadas as taxas de declínio do pico de produção até o final da última colheita e a taxa média de declínio para os três tratamentos. Para o T1 a taxa de declínio foi de $44,78 \%$, maior que a observada para o T2 e T3, de 29,64 e $32,01 \%$, respectivamente, indicando que o rbST diminui a taxa de queda da produção de leite após o pico de produção.

Gallo et al. (1997) atribuíram o aumento de $40 \%$ na produção de leite em cabras tratadas com
Tabela 10 - Constantes estimadas da curva de produção de leite ajustada pela função gama

Table 10 - Estimated constants for the milk production curve adjusted bymeans of the gamma function

\begin{tabular}{lccc}
\hline $\begin{array}{l}\text { Tratamento } \\
\text { Treatment }\end{array}$ & $\mathrm{A}$ & $\mathrm{B}$ & $\mathrm{C}$ \\
\hline 1 & 2,18348 & 0,14028 & 0,05330 \\
2 & 1,82634 & 0,04814 & 0,02706 \\
3 & 2,11397 & 0,10372 & 0,03608 \\
\hline
\end{tabular}

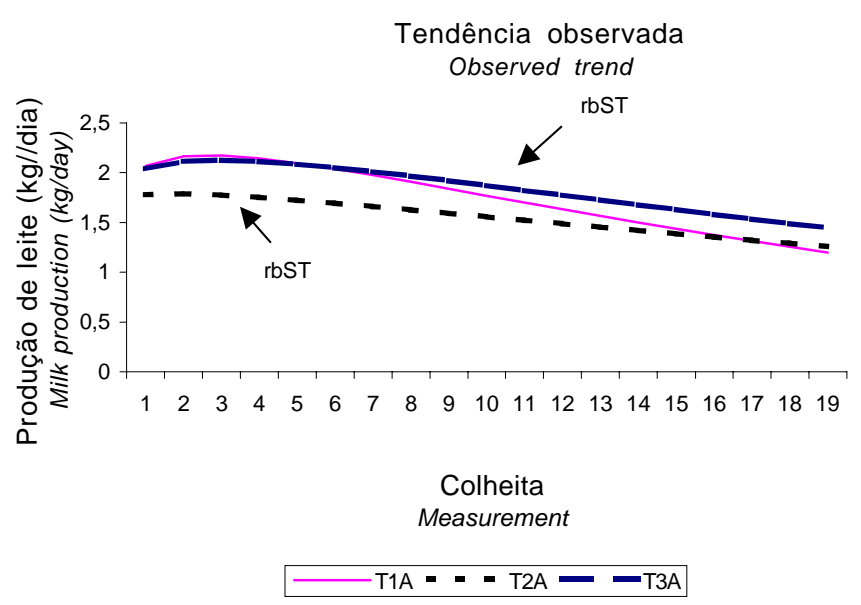

Figura 5 - Tendência ajustada pela função gama (média de mínimos quadrados) da produção de leite por tratamento, em função da coleta.

Figure 5 - Gamma-adjusted milk trend (least squares means), according to the treatment and measurement.

rbST ao aumento da persistência da lactação. Esse aumento da persistência poderia ser devido à capacidade desse hormônio em limitar a redução do parênquima mamário, à medida que a lactação progride ou à modulação do sistema plasmino-plasminogênio, conforme sugerido por Baldi (1999).

\section{Constituintes do leite}

Na Tabela 12 é apresentado o resumo da análise de variância dos constituintes do leite. Não foi observada influência de tratamento $(\mathrm{P}>0,01)$ sobre densidade, teor de gordura, extrato seco total (EST), lactose e proteína do leite.

Estes resultados estão de acordo com os de Baldi (1999), em que cabras Saanen tratadas com rbST, porém em fase final de lactação, não apresentaram alteração na porcentagem de gordura. Concordam em parte com Chadio et al. (2000), que trabalharam com oito cabras multíparas lactantes, 
Tabela 11 - Médias da produção de leite por tratamento ajustada pela função gama Table 11 - Treatment means of milk production adjusted by means of the gamma function

\begin{tabular}{|c|c|c|c|}
\hline \multicolumn{4}{|l|}{ Measurement } \\
\hline 1 & 2,07 & 1,78 & 2,04 \\
\hline 2 & 2,16 & 1,79 & 2,11 \\
\hline 3 & 2,17 & 1,78 & 2,13 \\
\hline 4 & 2,14 & 1,75 & 2,11 \\
\hline 5 & 2,10 & 1,72 & 2,09 \\
\hline 6 & 2,04 & 1,70 & 2,05 \\
\hline 7 & 2,00 & 1,66 & 2,01 \\
\hline 8 & 1,91 & 1,63 & 1,97 \\
\hline 9 & 1,84 & 1,59 & 1,92 \\
\hline 10 & 1,77 & 1,56 & 1,87 \\
\hline 11 & 1,70 & 1,52 & 1,82 \\
\hline 12 & 1,63 & 1,49 & 1,77 \\
\hline 13 & 1,56 & 1,45 & 1,73 \\
\hline 14 & 1,50 & 1,42 & 1,68 \\
\hline 15 & 1,44 & 1,39 & 1,63 \\
\hline 16 & 1,37 & 1,35 & 1,58 \\
\hline 17 & 1,31 & 1,32 & 1,54 \\
\hline 18 & 1,25 & 1,29 & 1,49 \\
\hline 19 & 1,20 & 1,26 & 1,45 \\
\hline Redução do pico ao último controle (\%) & 44,78 & 29,64 & 32,01 \\
\hline \multicolumn{4}{|l|}{ Reduction from peak to last measurement (\%) } \\
\hline Redução média por coleta $(\%)$ & 2,79 & 1,74 & 2,00 \\
\hline Mean decrease (\%) by measurement & & & \\
\hline
\end{tabular}

Tabela 12 - Resumo da análise de variância para os constituintes do leite Table 12 - Summary of the analysis of variance for milk constituents

\begin{tabular}{|c|c|c|c|c|c|c|}
\hline \multirow[t]{2}{*}{$\begin{array}{l}\text { Fonte de variação } \\
\text { Source of variation }\end{array}$} & \multirow[t]{2}{*}{$\begin{array}{l}\text { Graus de liberdade } \\
\text { Degrees of freedom }\end{array}$} & \multicolumn{5}{|c|}{$\begin{array}{l}\text { Quadrado médio } \\
\text { Mean square }\end{array}$} \\
\hline & & $\begin{array}{c}\text { Densidade } \\
\text { Density }\end{array}$ & $\begin{array}{c}\text { Gordura } \\
\text { Fat }\end{array}$ & $\begin{array}{l}\text { EST } \\
T S E\end{array}$ & $\begin{array}{l}\text { Lactose } \\
\text { Lactose }\end{array}$ & $\begin{array}{c}\text { Proteína } \\
\text { Protein }\end{array}$ \\
\hline $\begin{array}{l}\text { Tratamento } \\
\text { Treatment }\end{array}$ & 2 & 0,00000297 & 1,4750 & 1,9629 & 0,1300 & 0,4193 \\
\hline $\begin{array}{l}\text { Erro }(\mathrm{A}) \\
\operatorname{Error}(A)\end{array}$ & 12 & 0,00000925 & 1,3138 & 2,7549 & 0,5881 & 0,6342 \\
\hline $\begin{array}{l}\text { Coleta } \\
\text { Measurement }\end{array}$ & 10 & $0,00001294 * *$ & $1,7925^{* *}$ & $3,3509 * *$ & $2,1542 * *$ & $2,7382 * *$ \\
\hline $\begin{array}{l}\text { Tratamento } \\
\text { x Coleta }\end{array}$ & 20 & 0,000000921 & 0,2003 & 0,5023 & 0,1128 & 0,0967 \\
\hline $\begin{array}{l}\text { Treatment x Measurement } \\
\text { Erro(B) } \\
\text { Error }(B)\end{array}$ & 111 & 0,000001284 & 0,1206 & 0,4753 & 0,1117 & 0,0971 \\
\hline
\end{tabular}

$\mathrm{EST}=$ extrato seco total $(T S E=$ total solids extract $)$.

** $(P<0,01)(P<.01)$.

em condições similares de ordem de parição e tempo de lactação, e não observaram diferença significativa entre cabras tratadas e não tratadas quanto à porcentagem de proteína do leite, porém a porcentagem de gordura e lactose foi maior no grupo tratado. O maior conteúdo de lactose no leite das cabras tratadas foi atribuído ao aumento de glicose nas células mamárias, ligado às propriedades diabetogênicas do $\mathrm{GH}$.

Aumentos nos teores de gordura e lactose foram observados por Chadio et al. (2000).

A coleta apresentou efeito $(\mathrm{P}<0,01)$ sobre todos os constituintes do leite (Tabela 12).

Na Figura 6, são apresentadas as médias para densidade, gordura, EST, lactose e proteína do leite de cabra durante o período experimental. 
De maneira geral, a densidade do leite apresentou ligeiro aumento com o avanço das coletas ou progresso da lactação.

O teor médio de gordura e proteína do leite apresentaram-se conforme o esperado, ou seja, em ordem inversa a curva de produção de leite. No pico de produção de leite, observaram-se os menores valores de gordura e proteína e, à medida que a produção de leite declinou, houve aumento dos teores (Figuras 5 e 6 ).

Os teores médios de lactose do leite apresentaram tendência de queda a partir da primeira até a ultima coleta (Figura 6).

De maneira geral, as tendências das curvas dos constituintes do leite ao longo da lactação não foram diferentes do relatado na literatura (Aguirre, 1986) para lactações normais.

A última coleta de leite foi prejudicada, pois alguns animais já haviam secado espontaneamente e outros já apresentavam um leite de coloração amarelada, com aspecto de colostro. Possivelmente, por este motivo, houve uma diferença tão grande entre a coleta 11 e as demais.

Viabilidade econômica do uso do rbST

Para a viabilidade do uso do rbST foram considerados os seguintes parâmetros: preço de venda do leite na safra de $R \$ 1,50$ e na entressafra de $R \$ 2,00$; custo do concentrado $\mathrm{R} \$ 0,30$, sendo fornecido $1 \mathrm{~kg}$ para cada 2,5 kg de leite; custo da aplicação do rbST por cabra $\mathrm{R} \$ 2,10$ (a cada 14 dias).

Na Tabela 13 pode-se verificar que, na safra, o tratamento com rbST custa o equivalente a $1,52 \mathrm{~L}$ de leite, ou seja, em um período de 14 dias os animais tratados deveriam produzir, em média, $110 \mathrm{~g}$ de leite/dia a mais para que o tratamento fosse economicamente viável. Na entressafra, essa produção seria de 1,12 L de leite em 14 dias ou $80 \mathrm{~g}$ de leite/dia.

Dessa forma, a utilização do rbST pode ser uma opção viável ao produtor rural que, por algum motivo de mercado, precise aumentar a persistência da produção de leite durante certo espaço de tempo.

\section{Características reprodutivas}

As características reprodutivas do parto no início do experimento e do parto após o experimento são apresentadas na Tabela 14.

Embora sem análise estatística, uma vez que o número de informações é pequeno em se tratando de características reprodutivas, parece que os animais do T2 e T3 apresentaram fertilidade menor que T1. Esse fato mereceria ser melhor avaliado com maior quantidade de informações.

Tabela 13 - Viabilidade econômica da utilização do rbST em cabras lactantes

Table 13 - Economic analysis by using rbST in lactating goats

\begin{tabular}{lcc}
\hline $\begin{array}{l}\text { Itens } \\
\text { Item }\end{array}$ & $\begin{array}{c}\text { Safra } \\
\text { High production season }\end{array}$ & $\begin{array}{c}\text { Entressafra } \\
\text { Low production season }\end{array}$ \\
\hline $\begin{array}{l}\text { Preço de venda do leite }(\mathrm{R} \$) \\
\text { Milk selling price }(R \$)\end{array}$ & 1,50 & 2,00 \\
$\begin{array}{l}\text { Custo do concentrado por litro de leite produzido }(\mathrm{R} \$) \\
\text { Cost of concentrate/produced liter of milk }(R \$)\end{array}$ & 0,12 \\
$\begin{array}{l}\text { Custo do rbST por animal }(\mathrm{R} \$) \\
\text { Cost of rbST/animal }(R \$)\end{array}$ & 2,10 \\
$\begin{array}{l}\text { Preço de venda do leite descontando o concentrado }(\mathrm{R} \$) \\
\text { Milk selling price minus concentrate cost }(R \$)\end{array}$ & 1,38 \\
$\begin{array}{l}\text { Relação custo rbST/preço líquido do leite }(\mathrm{L}) \\
\text { Ratio of rbST cost:net price milk }(L)\end{array}$ & 1,52 & 2,10 \\
\hline
\end{tabular}


Tabela 14 - Características reprodutivas de cabras leiteiras submetidas ao tratamento com rbST Table 14 - Reproductive traits of the dairy goats treated with rbST

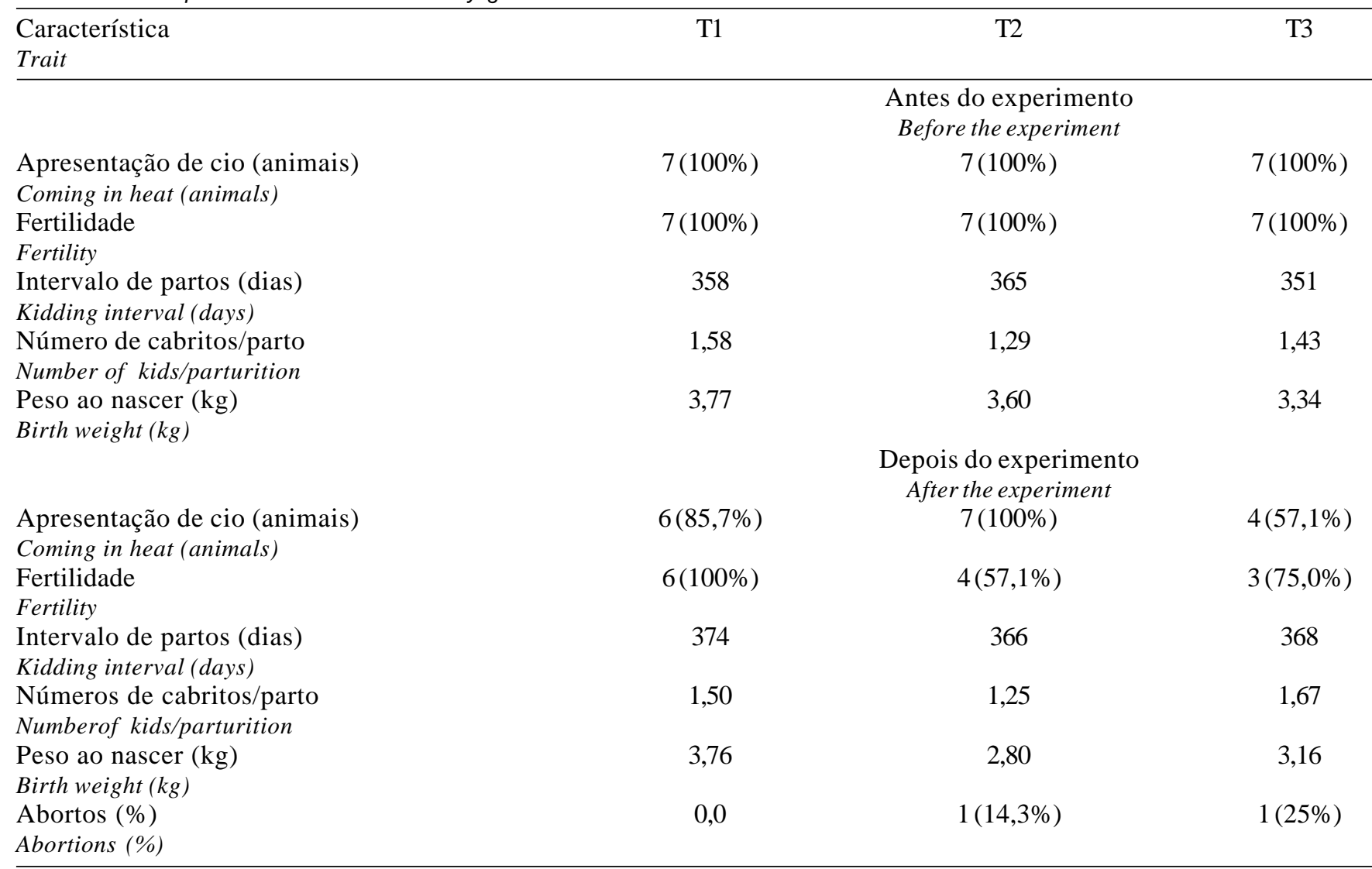

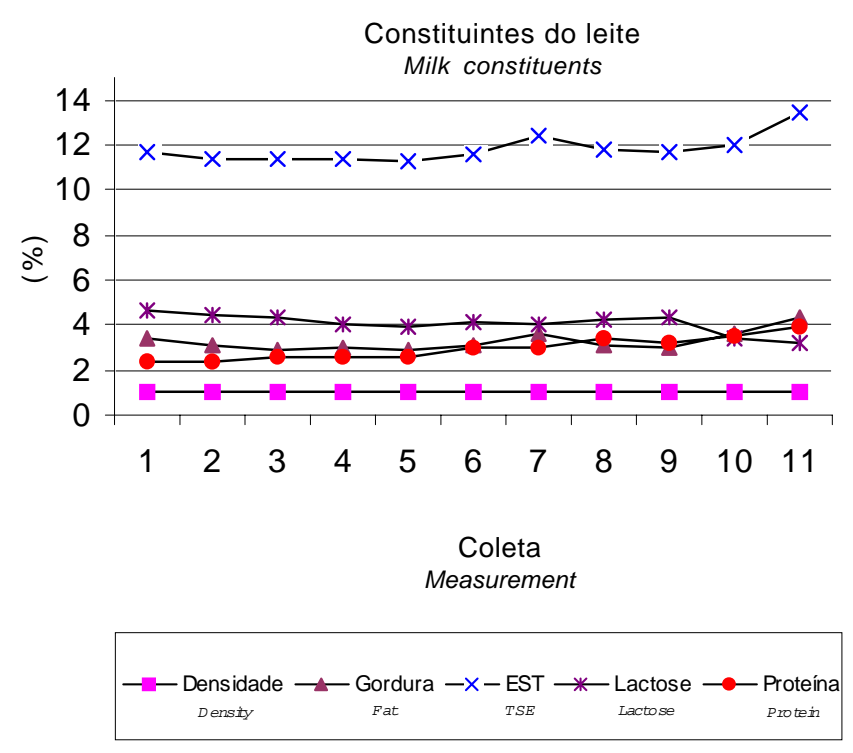

Figura 6 - Constituintes do leite, em função de coletas. Figure 6 - Milk constituents, according to the measurements.

\section{Conclusões}

Cabras tratadas com rbST não apresentaram aumento efetivo na produção de leite, porém a persistência da lactação foi melhorada com a aplicação do hormônio.

O peso e o índice de condição corporal não foram afetados pelo uso do rbST, indicando que a mobilização de reservas corporais, quando acontece, é pouco perceptível e os animais acabam se adaptando ao uso do rbST.

Os constituintes do leite não foram afetados pelo tratamento com rbST e apresentaram-se com valores normais ao longo da lactação.

O tratamento com rbST pode ser uma opção economicamente viável ao produtor rural, uma vez que a relação entre o custo do produto e o preço de venda do leite é baixo, principalmente na entressafra. 


\section{Literatura Citada}

AGUIRRE, S.I.A. Productos caprinos. In: AGUIRRE, S.I.A. (Ed.) Produccion de caprinos. 1.ed. México: AGF Editor, 1986. p.105-178.

ASSOCIATION OF OFFICIAL ANALYTICAL CHEMISTS AOAC. Official methods of analysis. 12.ed. Washington, D.C., 1975. 1094p.

BALDI, A. Manipulation of milk production and quality by use of somatotropin in dairy ruminantes other than cow. Domestic Animal Endocrinology, v.17, p.131-137, 1999.

BARIL, G.; REMY, B.; LEBOEUF, B. et al. Synchonization of estrus in dairy goats: the relationship between eCG binding plasma, time of occurrence of estrus and fertility following artificial insemination. Theriogenology, v.45, p.1553-1559, 1996.

CHADIO, S.E.; ZERVAS, G.; KIRIAKOU, K. et al. Effects of recombinant bovine somatotropin administration to lactation goats. Small Ruminant Research, v.35, p. 263-269, 2000.

CORTEL, J.M.; LEBOEUF, B. Evolution technico-économique de I'insémination artificielle caprine. Elevage \& Insémination, v.237, p.3-17, 1990.

CORTEL, J.M.; LEBOEUF, B.; BARIL, G. Artificial breeding of goats and kids induced to ovulate with hormones outside the breeding season. Small Ruminant Research, v.1, p.19-35, 1988.

CROSCLAUDE, F.; RICORDEAU, G.; MARTIN, P. et al. Du gène au fromage: le polymorphisme de la caséine aS 1 caprine, ses effects, son évolution. INRA Production Animal, v.7, n.1, p.3-19, 1994.

DISENHAUS, C.; JAMMES, H.; HERVIEU, J. et al. Effects recombinant bovine somatotropin on goat milk yield, composition and plasma metabolites. Small Ruminant Research, v.15, p. 139-148, 1995.

FURTADO, M.M. Caracterização eletroforética das caseínas alfa e beta do leite de cabra. Revista do Instituto de Laticínios Cândido Tostes, v.38, n.226, p.27-29, 1983.

GOMES, F.P. Curso de estatística experimental. 10.ed. Piracicaba: Nobel, 1982. 430p.

GALLO, L.; BAILONI, L.; SCHIAVON, S. et al. Effect of sowrelease somatotropin on the pattern of milk yield between and within injection intervals. Journal of Dairy of Dairy Science, v.80, p. 46-51, 1997.

INSTITUT NATIONAL DE LA RECHERCHE AGRONOMIQUE - INRA. Alimentacion de bovins, ovins \& caprins. Madrid: Mundi-Prensa, 1990. 432p.

INSTITUTO ADOLFO LUTZ. Métodos químicos e físicos para análise de alimentos. Normas analíticas. 3.ed. São Paulo, 1985. v.1, 533p.

McDOWELL, G.H., GOODEN, J.M., LEENANURUSKA, M.J. et al. Effects of exogenous growth hormone on milk production and nutrient uptake by muscle and mammary tissues of dairy cows in mid-lactation. Australian Journal of Biological Sciences, v.40, p.295-306, 1987.

MEPHAM, T.B., LAWRENCE, S.E., PETERS, S. et al. Effects of exogenous growth hormone on mammary function in lactating goats. Hormone and Metabolism Research, v.16, p.248-253, 1984.
MORAND-FEHR, P., HERVIER, J. Notaction de l'etat corpore: a vos stylos! La Chevre, v.175, p.28-31, 1989.

MOUCHRECK, E., MOULIN, C.H.S. Comportamento sexual de fêmeas caprinas sem raça definida (SRD) no estado de Minas Gerais. Informe Agropecuário, v.13, p.3-8, 1987.

NATIONAL RESEARCH COUNCIL - NRC. Nutrient requirements of goats. 3.ed. Washington, D.C.: 1981.91p.

NIELSEN, M.O. Effect of recombinantly derived bovine somatotropin on mammary gland synthetic capacity in lactating goats. Journal of Animal Physiology and Animal Nutrition, v.59, p.263-272, 1988.

PROSSER, C.G., MEPHAM, T.B. Mechanism of action of bovine somatotropin in increasing milk secretion in dairy ruminants. In: SEJRSEN, K.; VERSTERGAAD, M., NEIMANN-SORENSEN, A. (Ed.) Use of somatotropin in livestock production. London: Elsevier Applied Science. 1989. p.1-17.

PROSSER, C.G., ROYLE, C., FLEET, I.R. et al. The galactopoietc effect of bovine growth hormone in goats is associated with increased concentrations of insulin-like growth factor-I in milk and mammary tissue. Journal of Endocrinology, v.128, p.457-463, 1991.

RODRIGUES, M.A. Efeito da manipulação do fotoperíodo na indução de estro em cabras leiteiras mestiças. Viçosa, MG: Universidade Federal de Viçosa, 1992. 65p. Dissertação (Mestrado em Zootecnia) - Universidade Federal de Viçosa, 1992.

SAUVANT, D., MORAND-FEHR, P. Energy requirements and allowances of adult goats. In: MORAND-FEHR, P. (Ed.) Goat nutrition. Wageningen: Pudoc, 1991. p.61-72.

SILVA, D.J. Análise de alimentos (Métodos químicos e biológicos). 2.ed. Viçosa, MG: Universidade Federal de Viçosa, 1990. $165 \mathrm{p}$.

SODERHOLM, C.G.; OTTERBY, D.E.; LINN, J.G. et al. Effects of recombinant bovine somatotropin on milk prodution, body composition and physiological parameters. Journal of Dairy Science, v.71, p.355-365, 1988.

UNIVERSIDADE FEDERAL DE VIÇOSA-UFV. SAEG Sistema de análises estatísticas e genéticas. Versão 7.1. Viçosa, MG, 1997. 150p. (Manual do usuário).

WEISS, W.P.; CONRAD, H.R.; PIERRE, N.R.S. A theoretically based model for predicting total digestible nutrient values of forages and concentrates. Animal Feed Science and Technology, v.39, 95-100, 1992.

WOOD, P.D.P. Algebraic model of the lactation curve in cattle. Nature, v.216, p.164-165, 1967. 